PHYSICAL REVIEW D 93, 089910(E) (2016)

\title{
Publisher's Note: Leptophilic dark matter in Galactic Center excess [Phys. Rev. D 93, 083504 (2016)]
}

Bo-Qiang Lu and Hong-Shi Zong

(Received 21 April 2016; published 27 April 2016)

DOI: 10.1103/PhysRevD.93.089910

This paper was published online on 6 April 2016 with an incorrect source listing in Ref. [10]. Reference [10] should read as "The Fermi-LAT Collaboration, arXiv:1511.02938." The reference has been corrected as of 22 April 2016. The reference is correct in the printed version of the journal. 